consequences worsening EQ may have for cardiometabolic health and health inequities in this population.

Methods Using longitudinal data on employment stability, material rewards, workers' rights, working-time arrangements, unionization, and interpersonal power relations from the Health and Retirement Study, we used principal component analysis to construct an EQ score. Then, we used sequence analysis to identify late-career EQ trajectories (age 50-70 years; $\mathrm{N}=11,958$ respondents), overall and by race, gender, educational attainment, marital status. Finally, we estimated the association between trajectory-membership and postsequence-analysis-period prevalence of poor cardiometabolic health.

Results We identified ten EQ trajectories; the most prevalent trajectories were Minimally Attached and Wealthy (13.9\%) and Good EQ to Good Retirement (13.7\%), however, $42 \%$ of respondents were classified into suboptimal trajectories. Those in suboptimal trajectories were disproportionately women, Hispanic and/or Black, Indigenous and People of Color, and those with lower educational attainment. Moreover, those in suboptimal trajectories tended to report the worse cardiometabolic health. For example, the prevalence of hypertension was lowest for those in the Wealthy Business Owners trajectory and highest for Workers with Premature Mortality, followed by workers with Fair EQ and Good but Diminishing Wealth in Retirement.

Conclusion EQ is inequitably distributed and may play a role in cardiometabolic health inequities in later life.

\section{S-214 THE WORKING LIVES OF GENERATION Y: APPROACH AND CHALLENGES}

${ }^{1}$ Karin Veldman, Sander van Zon, Iris Arends, Ute Bültmann. ${ }^{1}$ University Medical Center Groningen, Netherlands

\subsection{6/OEM-2021-EPI.421}

Objective Generation $\mathrm{Y}$ has to earn a living in a new world of work in changing labor markets. To date, little is known about the working lives of todays' young adults and how early life factors impact their working lives. The aim of this study is to examine how educational and work factors cluster in young workers and to assess the impact of mental health problems from childhood to young adulthood on the worklife clusters.

Methods Longitudinal data of $\mathrm{N}=1235$ participants of the Tracking Adolescents Individual Life Study (TRAILS), a prospective cohort study from age 11 to 26, was used. To examine the participants' clusters regarding educational attainment, employment status, social benefits, type of contract, and working hours at age 26, latent class analysis was performed. Trajectories of mental health problems measured at ages 11, 13.5, 16, 19 and 26 were identified with latent class growth models. Multinomial logistic regression analyses were used to examine the associations between mental health and work-life clusters.

Results Five clusters were identified: 1) high educated fulltime workers $(30.6 \%), 2)$ medium educated part-time and fulltime workers $(21.6 \%), 3)$ students and medium educated workers with small jobs $(26.0 \%), 4)$ fulltime students (15.7\%), and 5) social benefit recipients (6.0\%). Participants with high-stable trajectories of internalizing and externalizing problems had an increased risk of receiving social benefits (OR 9.80, 95\%CI 4.26-22.5 and OR 13.9, 95\%CI 4.1546.9, respectively).

Conclusion At age 26, five work-life clusters were identified, showing the diversity of the working lives of today's young adults. The results of this study also show the long-term consequences of early mental health problems on the working lives of young adults. During the presentation, conceptual and analytical challenges will be discussed, for example: 'When does employment begin' and 'How can we best capture the new world of work?'

\section{S-215 WORKING LIFE, HEALTH AND WELL-BEING OF PARENTS: A JOINT EFFORT TO UNCOVER HIDDEN TREASURES IN BIRTH COHORTS}

${ }^{1}$ Milena Maule, Monica Ubalde-Lopez, Tina Garani-Papadatos, Ghislaine Scelo, Maribel Casas, Claudia Lissaker, Susan Peters, Ellen Aagaard Nohr, Raquel Lucas, Maria Albin, Kyriaki Papantoniou, Kinga Polańska, Cecilia H Ramlau-Hansen, Jelena Sarac, Jenny Sela. 'University of Turin, Italy

\subsection{6/OEM-2021-EPI.422}

Introduction Birth cohorts collect information on employment and health of parents (especially mothers) before and during pregnancy, at birth, and often after birth. These valuable and under-utilized data are typically collected for measuring their effects on the health of the children but have the potential to address specific domains difficult to assess in traditional occupational epidemiology studies, such as working life in relation to reproductive life and work-family conflicts.

Objectives We examine how birth cohort data could be exploited to study the bidirectional relationship and interactions between parenthood, work, and health in parents themselves, taking a life-course approach.

Methods Using a web-based database of birth cohorts, we summarize information on maternal employment and health conditions and other potentially related variables in cohorts spread throughout Europe. This provides information on what data are available and could be used in future studies, and what is missing if specific questions need to be addressed, exploiting the opportunity to explore work-health associations across heterogenous geographical and social contexts.

Results We highlight the many potentialities provided by birth cohorts and identify gaps that need to be addressed to adopt a life-course approach and investigate topics specific to the peripregnancy period, such as psychosocial aspects. We address the technical difficulties implied by data harmonisation and the ethical challenges related to the repurposing of data, and provide scientific, ecological and economic arguments in favour of improving the value of data already available as a result of a serious investment in human and material resources.

Conclusions There is a hidden treasure in birth cohorts that deserves to be brought out to study the relationships between employment and health among working parents in a time when the boundaries between work and life are getting stretched more than ever before. 